\title{
Poster \\ Digital Asset Management Systems: Open Source or Not Open Source?
}

\author{
Marina Morgan \\ Florida Southern College, \\ United States \\ mmorgan@flsouthern.edu
}

\author{
Naomi Eichenlaub \\ Ryerson University, \\ Canada \\ neichenl@ryerson.ca
}

Keywords: digital asset management system; content management system; web content management system; open source; proprietary; metadata.

\begin{abstract}
The objective of this poster is to provide an overview of a number of existing open source and proprietary information management systems for digital assets. We hope that this poster will assist libraries and other institutions in their process of researching and decision-making when considering implementing a management system for their digital collections.
\end{abstract}

\section{Background}

It should be noted that while neither of the authors is currently involved in a digital asset management system migration or selection project, they have a working knowledge of all the systems described herein. Additionally, in gathering data for this project it became clear that some of the systems evaluated here have been implemented as institutional repositories as well. However, this is beyond the scope of this poster. The Digital Asset Management systems chosen for the purpose of this poster were evaluated based on their capabilities of managing a collection of digital assets such as images, videos, sound recordings, and other multimedia content.

Moreover, from the beginning it became paramount to have a clear distinction between the different terminologies used: digital asset management systems (DAM) and content management systems or web content management system (CMS). While content management systems were built to allow non-technical users to create, publish and manage website content, digital asset management systems provide an infrastructure for management and preservation of digital assets.

\section{Introduction}

As the volume of digital resources owned or created increases, many institutions want to adopt a single platform with robust functionalities for discovery, storage, and cataloging of resources. According to The National Initiative for a Networked Cultural Heritage (NINCH), "Digital Asset Management (DAM) systems provide the means to manage digital assets from creation to publication and archiving". DAM systems have become a core part of the institutions' infrastructure using rich metadata as the basis for enhanced resource discovery as well as for use in teaching and learning. These days, choosing a DAM solution invariably means choosing either an open source or a proprietary solution. Open source software has source code that is publicly available so that it can be copied, modified, and redistributed royalty-free (though usually with attribution in the form of some type of Creative Commons license) (Fitzgerald, B., Kesan, J.P. \& Russo, B., 2011). The code is developed and maintained by communities of practice. Proprietary software on the other hand, is locked down in terms of access to code and made available for a fee from commercial enterprises. Unfortunately, there are no perfect products that offer off-theshelf solutions to all the unique needs of each institution. However, there are systems that are appropriate for specific kinds of collections, as showcased below. 


\section{Methodology}

For the purpose of this poster we chose three open source and three proprietary DAM systems for digital collections. They were selected based on functionality, packages and frameworks, ease of installation, number of users, scalability, metadata schemas and formats, hosting options, and technical support. Based on these characteristics, the open source DAMs reviewed are Islandora, Omeka, and DSpace, while the proprietary ones are CONTENTdm, Shared Shelf, and Digital Commons. Information was gathered by reviewing relevant literature on the topic of managing digital collections with a particular focus on collections and digital assets management systems.

\section{Open Source DAMs}

\subsection{Islandora}

a. Functionality. Born at the University of Prince Edward Island Library, Islandora is built on a software stack of FedoraCommons (repository layer), Islandora (integration layer) and Drupal (user interface layer) alongside Solr search (Ruest \& Stapelfeldt, 2014). A highly extensible open-source software framework, Islandora does not have default functionality (i.e. indexing, discovery, delivery) but instead allows developers to build their own or integrate third-party options into its framework (Castagné 2013).

b. Technical Summary. Islandora uses 'solution packs' which have evolved as "best-practice workflows" from the Islandora community's experience dealing with a variety of data types (https://wiki.duraspace.org/display/ISLANDORA715/About+Islandora). Islandora excels at preserving the integrity of collections and can be customized to manage any digital asset.

c. Metadata Standards and Formats. Islandora uses MODS metadata format and generates a DC version each time the MODS is modified. Islandora generates PREMIS XML metadata on demand. You can create custom XML metadata forms as well.

\subsection{Omeka}

a. Functionality. Omeka is an open source web-publishing platform developed by the Roy Rosenzweig Center for History and New Media at George Mason University (https://omeka.org/). There are two options for using Omeka: you can install Omeka using LAMP (Linux, Apache, MySQL and PHP) as a self-hosted option or you can sign up for the hosted Omeka.net solution. The former requires more technical expertise and access and allows for more customization while the latter is more plug-and-play. A thorough comparison of Omeka.org vs Omeka.net is available at http://info.omeka.net/about/.

b. Technical Summary. Omeka can be populated using batch migration tools, ie. OAI-PMH (Open Archives Initiative Protocol for metadata Harvesting), CSV, EAD, or Zotero. The Omeka API allows for customizable web design and an extensive list of plugins has been developed by the Omeka community.

c. Metadata Standards and Formats. Omeka provides default metadata support for Dublin Core with a plugin for Dublin Core Extended and a METS export. Alternatively you can create your own customized metadata vocabulary.

\subsection{DSpace}

a. Functionality. DSpace is a cross-platform open source solution primarily used as an institutional repository platform. There are, however, a number of institutions using it as a digital asset management system (DAMs), for example the Swinburne Image Bank (http://images.swinburne.edu.au/). In terms of open source software applications for digital assets management in general, DSpace has the largest community of developers and installations and there is also now a hosted option available called DSpaceDirect. 
b. Technical Summary. DSpace offers full support for OAI-PMH and SWORD (Simple Webservice Offering Repository Deposit). The latest releases of DSpace (5.x) indicate that they include support for CRUD (Create, Read, Update, Delete), linked open data, enhancements to DOI support and ORCID integration.

c. Metadata Standards and Formats. DSpace supports Qualified Dublin Core metadata with export options to many other formats including METS, MODS, RDF and MARC or you can create a custom XML metadata schema.

\section{Proprietary DAMs}

\subsection{CONTENTdm}

a. Functionality. CONTENTdm is a proprietary digital collection management system hosted and supported by OCLC. Installation is provided by OCLC, thus allowing the user to focus on creating and managing the digital collections. CONTENTdm enables a branded design and customization of the library digital collection website without prior programming skills.

b. Technical Summary. CONTENTdm has a robust technical infrastructure. It is delivered as "software as a service" (SaaS), meaning that there is no need to allocate personnel or hardware to manage the digital collections. Some of the technical features of interest are the OCR Extension to generate full-text transcripts from image files, batch importing from tab-delimited files, OAIPMH harvesting, and operational support for incremental backups.

c. Metadata Standards and Formats. CONTENTdm can handle document, image, video and audio files of any kind. There is full control over the digital resources access, descriptions, and display. Moreover, the fully customized metadata fields maximizes user discovery of materials.

\subsection{Shared Shelf}

a. Functionality. Artstor's Shared Shelf Commons is an open-access library of images, a Web-based service for cataloging and managing digital collections, either as a stand-alone tool or as an add-on to the Artstor Digital Library.

b. Technical Summary. Collections are managed without local technical infrastructure or administration. They are discoverable and may be shared with other institutions or published to the Open Web via Shared Shelf Commons, the Digital Public Library of America (DPLA), or your own Omeka site. New projects can be created from existing templates or by copying a specific project and modifying the fields as needed or adding local labels. After uploading the media files, to manage the digital assets you can catalog a single record or multiple records at once using the Master Record feature. You can also export and import, update and create multiple assets using Excel.

c. Metadata Standards and Formats. Support for different media types includes video, audio, documents, and images. It also provides easy access and use of Getty vocabularies, AAT, TGN, and ULAN, which are integrated into Shared Shelf Names. Metadata templates are based on Dublin Core, VRA Core 4.0 which can be customized and extended, or samples from the Astronomy Visualization Metadata (AVM) or Darwin Core fields.

\subsection{Digital Commons}

a. Functionality. Digital Commons is mainly used as an institutional repository, but the Image Galleries service allows for its implementation as a digital asset management system as well. Users can use Digital Commons as a place to host all types of visual content, such as digitized archives, scanned historical documents, photographs, and other items of a visual nature.

b. Technical Summary. Digital Commons serves as an effective platform for long-term image collection preservation, enabling viewing and sharing of the collection. You can batch upload images, create dynamic slideshows, and embed them throughout the digital collection. Users can explore large, high resolution images with the use of the pan and zoom viewer that can be 
displayed both on desktop and mobile devices. No longer relying on the discontinued Google API, the new content carousels and slideshows have a flexible implementation. Another consideration is that while OAI-PMH is supported to expose data, it does not however harvest OAI data from other sites.

c. Metadata Standards and Formats. Any field may be mapped to a Dublin Core value, or a custom export label. Digital Commons Image Service supports various media formats (Flash/HTML5 audio and video, Quick Time audio and video, RealAudio and RealVideo, Windows Media audio and video, YouTube, Vimeo, public domain files such as Internet Archive streaming, and other rich media via embedded API. You can add additional media fields for multiple media types.

\section{Challenges}

There are challenges with adopting both open source and proprietary software and selecting one or the other will be guided by the circumstances of each institution and even each project. In terms of implementing open source, the software may be free but there will definitely be a significant investment of staffing resources, most likely in the form of technical expertise. Alternatively, there are now a number of options to outsource open source implementation and hosting. Finally, there are well-established communities of practice to provide technical support for all the open source options described above.

On the other side, implementing proprietary solutions may be feasible for libraries without an IT staff. However, one of the major drawbacks of a proprietary-software package is expense. Depending on the number of users, the licensing and installation fee can be fairly expensive especially in comparison to open source software. While out-of-the-box solutions are easier to adopt, they are not usually as adaptable to the constantly changing needs of the institutions.

\section{Conclusions}

Choosing the right software to manage your digital collections is subjective and depends on specific circumstances, users' needs, budget, and licensing preferences. There is no shortage of options when it comes to managing, implementing, and describing your digital collections. There are proprietary systems that can be easily purchased and implemented; others require extensive knowledge of technical frameworks or a steep learning curve to implement. The software that you will choose depends on many variables and there is no perfect system when it comes to budget, needs, requirements, and implementation. Making an informed decision and evaluating all the options available will bring you closer to a system that matches the majority of your requirements.

\section{References}

bepress. (2016). Digital Commons Reference Material and User Guides. http://digitalcommons.bepress.com/reference/ Boiko, B. (2002). Content management bible. Hungry minds: New York.

Castagné, M. (2013). Institutional repository software comparison: DSpace, EPrints, Digital Commons, Islandora and Hydra. https://open.library.ubc.ca/cIRcle/collections/42591/items/1.0075768

CONTENTdm (2016). Support and Training. https://www.oclc.org/support/services/contentdm.en.html

Digital Asset Management and Museums - An Introduction. http://canada.pch.gc.ca/eng/1442946637162

DSpace (2016). Quickstart Guide. Retrieved May, 2016 from http://www.dspace.org/quick-start-guide

DSpace (2016). Use Case Examples: Image Repository http://www.dspace.org/repository_type/32

Fitzgerald, B., Kesan, J.P. \& Russo, B. (2011). Adopting open source software: a practical guide. MIT Press.

Gkoumas, G. \& Lazarinis, F. (2015). Evaluation and usage scenarios of open source digital library and collection management tools. Program: Electronic Library and Information Systems, 49(3), 226-241. doi: 10.1108/PROG-092014-0070 
Goh, D., Chua, A., Khoo, D., Khoo, E., Mak, E. \& Ng, M. (2006), A checklist for evaluating open source digital library software. Online Information Review, 30(4), 360-379. doi:10.1108/14684520610686283

Islandora (2016). Documentation http://islandora.ca/documentation

Krishnamurthy, M. (2007). Open access, open source and digital libraries: A current trend in university libraries around the world. Program: Electronic Library and Information Systems, 42(1), 48-55. doi:10.1108/00330330810851582

Larsen, R. \& W. Howard. (2004) Knowledge Lost in Information: Report of the NSF Workshop on Research Directions for Digital Libraries (Chatham, Mass.: University of Pittsburg, School of Information Sciences, June 15, 2003), available online at www.digitalpreservation.gov/news/2004/knowledge_lost_report200405.pdf

NINCH Guide to Good Practice in the Digital Representation and Management of Cultural Heritage Materials, Chapter XIII: Digital Asset Management. (2003) http://www.nyu.edu/its/humanities/ninchguide/XIII/

Oguz, F. (2016). Organizational Influences in Technology Adoption Decisions: A Case Study of Digital Libraries. College \& Research Libraries, 77(3), 314-334. doi:10.5860/crl.77.3.314

Omeka (2016). Documentation http://omeka.org/codex/Documentation

Rath, L. (2016). Omeka.net as a librarian-led digital humanities meeting place. New Library World 117(3/4), 158-172. doi:10.1108/NLW-09-2015-0070

Reddy, R. \& Wladawsky-Berger, I. (2001). Digital libraries: Universal access to human knowledge. Arlington, VA: National Coordination Office for Information Technology Research and Development.

Ruest, N. \& Stapelfeldt, K. (2014). Introduction to Islandora. http://yorkspace.library.yorku.ca/xmlui/handle/10315/28006

Shared Shelf (2016). Knowledge Base http://support.sharedshelf.org/knowledge-base

Virtual Astronomy Multimedia Project. (2007). Astronomy Visualization Metadata Standard. http://www.virtualastronomy.org/avm_metadata.php

Yeh, S.T,, Reyes, F., Rynhart, J., \& Bain, P. (2016). Deploying Islandora as a Digital Repository Platform: a Multifaceted Experience at the University of Denver Libraries. D-Lib Magazine, 22(7/8), doi: 10.1045/july2016-yeh 